\title{
Maria Dario, André Salmon. Alle origini della modernità poetica, Venezia
}

\section{Anna Boschetti}

\section{Q OpenEdition}

1 Journals

\section{Edizione digitale}

URL: https://journals.openedition.org/studifrancesi/40216

DOI: 10.4000/studifrancesi.40216

ISSN: 2421-5856

\section{Editore}

Rosenberg \& Sellier

\section{Edizione cartacea}

Data di pubblicazione: 1 juillet 2004

Paginazione: 150-152

ISSN: 0039-2944

\section{Notizia bibliografica digitale}

Anna Boschetti, «Maria Dario, André Salmon. Alle origini della modernità poetica, Venezia», Studi Francesi [Online], 142 (XLVIII | I) | 2004, online dal 30 novembre 2015, consultato il 09 septembre 2021. URL: http://journals.openedition.org/studifrancesi/40216; DOI: https://doi.org/10.4000/studifrancesi. 40216

Questo documento è stato generato automaticamente il 9 septembre 2021.

\section{(c) 9 (i) $\Theta$}

Studi Francesi è distribuita con Licenza Creative Commons Attribuzione - Non commerciale - Non opere derivate 4.0 Internazionale. 


\title{
Maria Dario, André Salmon. Alle origini della modernità poetica, Venezia
}

\author{
Anna Boschetti
}

\section{NOTIZIA}

Maria Dario, André Salmon. Alle origini della modernità poetica, Venezia, Istituto veneto di scienze, lettere ed arti, 2001, pp. VII-273.

«Nous nous sommes rencontrés dans un caveau maudit / Au temps de notre jeunesse / Fumant tous deux et mal vêtus attendant l'aube»" ${ }^{1}$ : così Apollinaire rievoca, nel 1909, il suo incontro con André Salmon, avvenuto nel 1903 in un caffè del Quartiere Latino, dove le «soirées» organizzate dalla Plume avevano offerto ai due oscuri esordienti, poco più che ventenni, la possibilità di leggere i loro versi e di inserirsi nella piccola cerchia della bohème letteraria. «... notre amitié a été le fleuve qui nous a fertilisés» scrive ancora Apollinaire: quest'amicizia, che includerà poco dopo Marx Jacob e Picasso, ha un effetto fecondante. Attraverso il continuo confronto e scambio, negli incontri quotidiani al Bateau-Lavoir, nascono esperienze che hanno un ruolo decisivo nella rivoluzione letteraria e artistica compiuta dall'avanguardia nel decennio che precede la prima guerra mondiale.

Dei tre poeti che si ritrovano nell'atelier di Picasso, Salmon è il primo a essere notato e apprezzato. La sua prima raccolta, Poèmes (1905), gli procura giudizi lusinghieri da parte di contemporanei esigenti e competenti, molto autorevoli nel circuito ristretto più attento all'innovazione, come Jarry, Moréas, Golberg, e il critico del Mercure de France, Pierre Quillard. Salmon apre la strada ad Apollinaire anche nel campo del giornalismo e della critica d'arte. Il suo libro, La Jeune peinture, precede di un anno la pubblicazione di quello di Apollinaire, Méditations esthétiques. Les Peintres cubistes. Salmon è anche autore di alcuni tentativi teatrali (scritti in collaborazione con 
Apollinaire) e di una serie di testi in prosa che, come quelli di Apollinaire e di altri poeti della loro generazione, mettono in discussione i modelli narrativi dominanti e le definizioni dei generi. Ma a differenza di Apollinaire e di Max Jacob, che sono consacrati tra i grandi del Novecento francese, oggi Salmon è trascurato dalla storia letteraria e ignorato dal grande pubblico. La maggior parte delle sue opere non sono state più ripubblicate. Solo recentemente gli sono stati dedicati studi approfonditi, come in particolare, quelli di Jaqueline Gojard, che ha dedicato un'analisi accurata a Le Manuscrit trouvé dans un chapeau, e di Marilena Pronesti, che si è occupata soprattutto della critica d'arte e del giornalismo.

3 Il libro di Maria Dario fornisce un importante contributo alla conoscenza dell'opera di Salmon e, più in generale, del posto che le spetta nella storia della poesia, poiché non si limita ad analizzare singoli aspetti, ma compie il lavoro di oggettivazione che è necessario per situare la traiettoria di Salmon e rendere intelligibili le sue scelte e il suo destino pubblico. L'unità di analisi che prende in considerazione non include semplicemente la poesia di Salmon, ma, da una parte, lo spazio in cui l'opera si è generata e si è progressivamente definita, e, dall'altra, il «punto di vista» del suo autore; non solo, dunque, l'opus operatum, i testi, ma anche il modus operandi, il modo di produzione dell'opera.

4 La «genesi» non è intesa qui soltanto nell'accezione ristretta (storia della redazione) che dà a questo concetto la critica "genetica», ma nel senso più ampio di processo collettivo che contribuisce a orientare e a modellare sia il «progetto creatore», sia la ricezione dell'opera. Maria Dario mostra che le opzioni tematiche e formali, se, soggettivamente, appaiono per lo più, agli autori stessi, come spontanee e indeterminate, sono sempre, oggettivamente, prese di posizione, modi di schierarsi rispetto alle potenzialità e alle sfide che ogni autore intravvede, secondo la posizione che occupa $\mathrm{e}$ secondo il grado in cui padroneggia il funzionamento del campo di produzione.

5 Per capire l'evoluzione della poesia di Salmon, Maria Dario la mette, dunque, in relazione con l'evoluzione del campo poetico, delle sue gerarchie implicite e della sua problematica, tra i primi anni del secolo e il 1914. Attraverso l'analisi di una singola traiettoria, questo lavoro, condotto con tutto il rigore, la finezza e le competenze diversificate che richiede la combinazione di focalizzazioni diverse, contribuisce, in tal modo, anche alla conoscenza di un periodo cruciale della storia letteraria.

6 I risultati mostrano la virtù euristica ed esplicativa di un simile approccio. Per la prima volta, si ha una visione d'insieme del percorso di Salmon fino alla guerra e del suo apporto al rinnovamento della scrittura poetica. Confrontando i testi (sottoposti ad un'analisi tematica e formale molto acuta e precisa) con i possibili della poesia, quali si presentano attraverso le opere e le altre prese di posizione dei contemporanei, Maria Dario fa emergere la singolarità e l'importanza delle ricerche di Salmon. Solo la comparazione, che individua le differenze rispetto ai modelli in circolazione, permette, in effetti, di misurare l'originalità della sua ispirazione, delle sue scelte metriche e della sua interpretazione del verso «libero».

7 L'analisi spiega, inoltre, una serie di aspetti dell'evoluzione di Salmon che, isolati, apparivano incomprensibili o contradditori. Il ritorno a posizioni più prudenti, nella terza raccolta (Le Calumet, 1910), dopo gli esperimenti innovatori proposti in Féeries (1907), diventa comprensibile, se vi si riconosce una reazione alla freddezza con cui la critica ha accolto questo secondo volume. Maria Dario mostra come un simile percorso sia controcorrente rispetto all'andamento della «borsa» dei valori poetici. Salmon 
appare precocemente «modernista», in una fase in cui la tendenza dominante è il ripiego su valori «sicuri»; all'opposto, egli rivendica la libertà del poeta di servirsi delle forme tradizionali, quando la questione della modernità è diventata la posta centrale della competizione all'interno della sua generazione.

Dal 1910 conduce una battaglia solitaria contro i gruppi (gli umanisti, i futuristi) che allora si fanno campioni di un modernismo dogmatico, opponendo loro un suo modernismo personale, troppo fuori dagli schemi per essere apprezzato nei suoi aspetti di novità. Si chiude, sempre più, in un aristocratico disprezzo verso le strategie manifesti, alleanze, riviste - con cui i suoi coetanei perseguono l'affermazione. Così, verso il 1912, è uno dei pochi che rimane isolato, nel momento in cui tutti i suoi principali concorrenti tendono a raggrupparsi e, pur divisi dalla rivalità, a riconoscere una problematica comune, che designano con una parola d'ordine (variamente interpretata): la «simultaneità».

9 La crisi del rapporto tra Salmon e Apollinaire si chiarisce anch'essa in questa visione strutturale, che fa vedere come il disaccordi non si possa ridurre a questioni personali, ma si fondi sulla divergenza delle traiettorie. Il primo si esclude, esce dalle Soirées de Paris (la rivista che hanno fondata, insieme con altri amici, all'inizio del 1912) e addirittura cessa di pubblicare poesia. Il secondo, persuaso che il poeta debba «appartenere alla sua epoca», si coinvolge nella competizione sempre più esasperata tra $\mathrm{i}$ «movimenti» che contribuiscono, attraverso le loro ricerche e i loro scontri, a trasformare radicalmente la nozione di poesia, e diventa il campione dell'avanguardia.

Come spesso avviene, in particolare quando si tratta di un'opera poliedrica, il percorso di Salmon attraversa più microcosmi, ognuno caratterizzato da una logica specifica: la poesia sperimentale, il giornalismo, l'arte d'avanguardia, la critica d'arte, l'editoria. Questa multiposizionalità presenta vantaggi e inconvenienti. Favorisce le strategie ibride, le opere sincretiche che sovente contribuiscono all'innovazione, mettendo in discussione i confini tra i generi, le categorie che modellano la percezione e i criteri di valutazione correnti. Maria Dario mette bene in luce l'importanza che hanno queste diverse esperienze, in particolare il confronto con i pittori e l'attività giornalistica. Ma non si può impunemente sfidare i principi di classificazione su cui si fondano le gerarchie. Anche per questo, come mostra l'analisi, le scelte di Salmon finiscono per sconcertare i contemporanei e apparire contraddittorie. Nel 1912 la sua poesia, a prima vista tradizionale, appare incompatibile con la posizione che prende come critico d'arte, difendendo le esperienze rivoluzionarie dei suoi amici pittori.

11 Attraverso l'esame di un caso particolare, questo libro contribuisce a verificare ipotesi generalizzabili e trasponibili, come esige il lavoro scientifico, utile al progresso della conoscenza. I risultati illustrano efficacemente come, superando la sterile contrapposizione tra lettura «interna» delle opere e ricostruzione «esterna» del processo di produzione, si possano rendere intelligibili le scelte più tecniche, apparentemente più personali e imprevedibili, e, al tempo stesso, si possa chiarire la genesi delle gerarchie letterarie. Maria Dario non si limita, infatti, a oggettivare i meccanismi che orientano l'evoluzione della poesia, ma prende in considerazione i fattori che intervengono nel riconoscimento.

12 Un'opera come quella di Salmon, che non rientra negli schemi dei contemporanei né in quelli dei posteri, porta a interrogarsi sui concetti che usiamo per parlarne. Nozioni come avanguardia, innovazione, originalità, modernità cessano di apparire scontate. Uno dei meriti dell'analisi consiste nel far vedere che dietro queste false evidenze ci 
sono significati profondamente diversi, e questi significati dipendono dal «punto di vista», cioè dalla posizione che si occupa, in uno spazio che è un prodotto storico, in costante trasformazione.

13 La storicizzazione non implica, tuttavia, una conclusione relativistica. Al contrario, essa mostra che le gerarchie non sono arbitrarie, ma si fondano sulle leggi tacite del campo di produzione. Le leggi fondamentali per la riuscita, in qualunque gioco sociale, sono il coinvolgimento nel gioco e la padronanza pratica delle regole che esso si è dato. La storia umana attesta che tutti i campi tendono a riconoscere come prove della grandezza la disponibilità al confronto e la capacità di sostenere le sfide che si delineano nella competizione. L'opera di Apollinaire non è meno «inclassificabile» di quella del suo amico Salmon, ed è più celebre che veramente conosciuta e compresa. Ma Apollinaire ha riconosciuto e affrontato tutte le sfide del suo tempo, Salmon non ha voluto o saputo fare altrettanto. Si è messo fuori dalla storia e non sorprende, allora, che possa rientrare nella storia solo grazie ad un'analisi retrospettiva, capace di far apparire il suo ruolo non marginale nella piccola «bande» che, all'alba del XX secolo, ha contribuito a cambiare l'arte e la poesia.

\section{NOTE}

1. G. APOllinaire, «Poème lu au mariage d'André Salmon», CEuvres poètiques, Paris, Gallimard, «Bibliothèque de la Pléiade», 1965, p. 83. 